\title{
Enhancing the sensitivity of interferometer sensing by slow light in a photonic crystal waveguide
}

\author{
Qi Wang, ${ }^{* 1,2}$ Bo Han, ${ }^{1}$ Chuan Qin, ${ }^{1}$ Kai Xue, ${ }^{1}$ and Yong Zhao ${ }^{1,2}$ \\ ${ }^{1}$ Northeastern University, College of Information Science and Engineering, Shenyang, China, \\ ${ }^{2}$ State Key Laboratory of Synthetical Automation for Process Industries (Northeastern University), Shenyang, China
}

Received October 29, 2012; accepted December 27, 2012; published December 31, 2012

\begin{abstract}
A novel high sensitivity Mach-Zehnder interferometer (MZI) with slow light in a photonic crystal waveguide (PCW) is proposed to enhance the sensitivity of a fiber Brag grating (FBG) sensing system. The output intensity of MZI would be changed with a reflect center wavelength change of the FBG, which relates to the applied strain. By designing the PCW parameters, the measuring sensitivity of the applied strain can reach up to $3.6 \times 10^{-3} \mathrm{rad} / \mu \varepsilon$ with a very high group index of 118 . The active region is only $0.1 \mathrm{~mm}$, which could be of great advantage in the use of limited space.
\end{abstract}

The present demodulation technology of a Mach-Zehnder interferometer (MZI) provides high precision and a simple method for the output signal of a fiber Brag grating (FBG) [1], which can be widely used to measure strain, temperature, and other physical and chemical parameters [2-4]. However, in many practical applications, sensitivity is a major constraining factor to its further development. During the past decade, slow light in photonic crystal waveguides (PCW) has been extensively studied to realize high sensitivity and a miniature sensor due to the advantages of working under room temperature, great potential bandwidth, and realizing slow light in an arbitrary wavelength [5-7].

In this paper, we propose a method to enhance the wavelength detection sensitivity of FBG by using an interferometer with slow light in PCW. The strain sensitivity can reach up to $3.6 \times 10^{-3} \mathrm{rad} / \mu \varepsilon$, and the size of this device is also decreased effectively.

As shown in Fig.1, FBG is adopted as a sensing front part, whose reflect center wavelength is changed with the applied strain. Therefore, we can get the change of the measured strain by detecting the change of the FBG center wavelength. To maintain the strain applied at every point of the FBG equality, the fiber grating is pasted on the central axis of the cantilever beam, which is an isosceles triangle [8]. When the strain occurs on the free end of the cantilever beam, the reflect center wavelength of FBG would change corresponding. Then the reflect signal is equally divided into the two arms of a Mach-Zehnder interferometer (MZI). The above one is the measuring arm which is composed of $\mathrm{PCW}$ and the below one is the reference arm which is an

\footnotetext{
*E-mail: wangqi@ise.neu.edu.cn
}

ordinary single mode optical fiber. The output lights would interfere with each other at the end of the two optical fibers and be detected by a photodetector. At last, the data would be processed and displayed by the computer. In this work, the interference phase of the two arms would be changed with a wavelength of input light, thus the strain of the cantilever beam could be obtained through detecting a change in output light intensity.

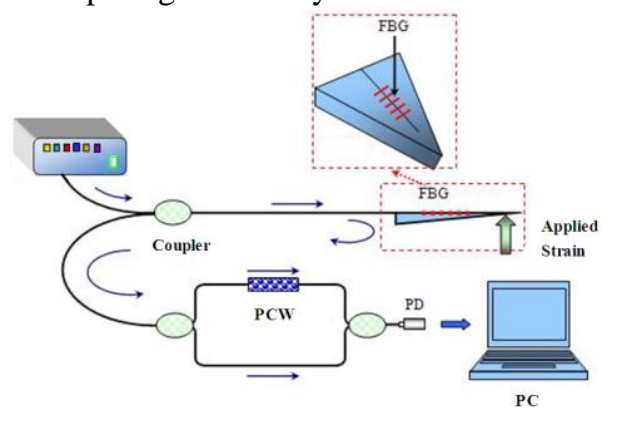

Fig.1. The structure of MZI for measurement of strain.

According to the interference theory, the output intensity of MZI is given by:

$$
I=\frac{I_{0}+I_{0} \cdot \cos (\Delta \varphi)}{2}
$$

where, $I_{0}, I$ are the input and output intensity of light respectively, the phase difference of the two arms can be expressed as: $\Delta \varphi(\lambda)=2 \pi n(\lambda) d \lambda^{-1}, d=0.1 \mathrm{~mm}$ is the path mismatch of the two arms and equals to the length of PCW in this paper, $n$ is the refractive index of PCW, which would be changed with the wavelength of input light $\lambda$.

We can further derive the wavelength change $\Delta \lambda$ of the FBG center wavelength using the equation:

$\frac{\mathrm{d} \Delta \varphi(\lambda)}{\mathrm{d} \lambda}=-\frac{2 \pi d}{\lambda^{2}} \cdot\left(n_{g}(\lambda)=n(\lambda)-\lambda \frac{\mathrm{d} n(\lambda)}{\mathrm{d} \lambda}\right)=-\frac{2 \pi d}{\lambda^{2}} \cdot n_{g}(\lambda)$.

The relationship between the reflective wavelength change of FBG and the strain of the cantilever beam is $\mathrm{d} \lambda=\gamma \cdot \mathrm{d} \varepsilon$, so the sensitivity of the interferometer sensor can be expressed by:

$$
\frac{\mathrm{d} \Delta \varphi(\lambda)}{\mathrm{d} \varepsilon}=-\frac{2 \pi d \gamma}{\lambda^{2}} \cdot n_{g}(\lambda)
$$

where $\varepsilon$ is the strain of the cantilever beam, $\gamma=1.15 \mathrm{pm} / \mu \varepsilon$ 
is the strain coefficient of FBG. In this system, PCW is applied to the measuring arm of MZI, thus the sensitivity of the interferometer could be greatly enhanced by an increase in the group index $n_{g}$. Naturally, the interferometer could detect a more precise change of the center wavelength of FBG and the measurement sensitivity of the strain is greatly improved, thus we should properly design the PCW structure to obtain an extremely high group index.

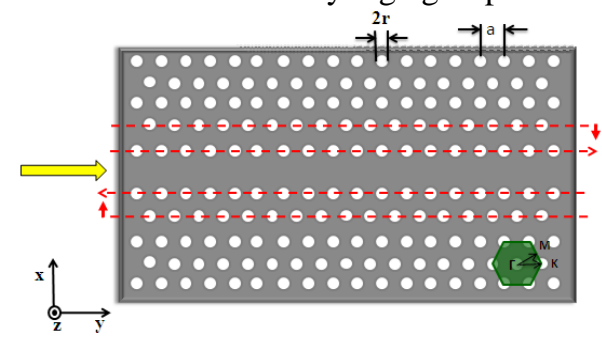

Fig.2. Structure of the proposed photonic crystal waveguide.

Figure 2 shows the configuration of PCW, which is constructed in a silicon slab $(n=3.48)$ by replacing the central row of air holes in the triangular lattice photonic crystal. The properties of PCW are numerically investigated by a two-dimensional (2D) plane wave expansion (PWE) with a slab equivalent index method and by selecting an appropriate supercell using the MIT photonic band (MPB) package [9], which is accurate enough to analyze the properties of PCW [10]. Due to the polarization sensitivity of 2D PhC only TE modes (electric fields are in the plane) are considered in this work [11]. The thickness of the slab $h=220 \mathrm{~nm}$, thus, the effective index of the slab of 2.87 is used in numerical simulation [12], and the radius of air holes $r=0.3 a$, where $a$ is the lattice constant.

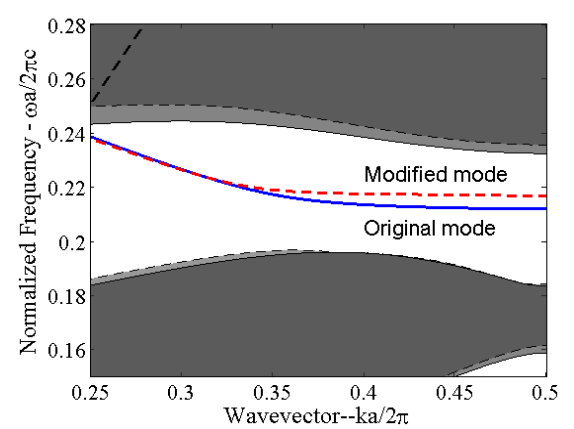

Fig. 3. Dispersion diagram of the designed and original PCW.

Inspired by Ref. [13], we design a procedure to generate large group index slow light in PCW by shifting the first row of air holes along the direction of the $y$ axe and the second row along the direction of the $x$ axis, and the movement directions along the arrows are positive. In this work, we design $d y=0.06 a$ and $d x=0.09 a$, which denote the shift distance of the air-holes along the directions of the $y$ and $x$ axes is $0.06 a$ and $0.09 a$, respectively.
Figure 3 shows the dispersion diagram of the TE mode in the designed structure, the dotted line indicated the guide mode, the slab mode region is marked with gray, and the dispersion diagram of the unmodified PCW with dashed line is also shown for comparison. It is clear that our modified guide mode possesses a flatter slope of the tail near the band edge, thus the group index $n_{g}=c \cdot(\mathrm{d} k / \mathrm{d} \omega)$ obtained from the slope of the dispersion curve can be greatly enhanced. Where $\omega$ is the frequency, $k$ is the wave vector, $c$ is the light velocity in vacuum. The group velocity dispersion (GVD) parameter $\beta$ is given by the second order derivative of the dispersion relation as: $\beta=\frac{\mathrm{d}^{2} k}{\mathrm{~d} \omega^{2}}=\frac{1}{c} \cdot \frac{\mathrm{d} n_{g}}{\mathrm{~d} \omega}$.

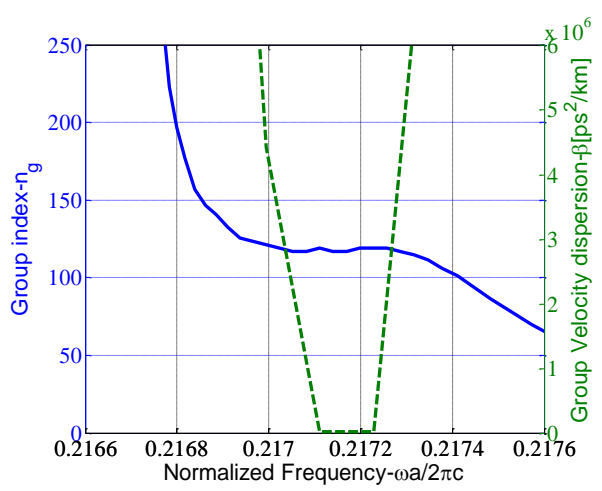

Fig. 4. Group index and group velocity curves of designed PCW.

Figure 4 is the group index and group velocity dispersion curves of our designed PCW, it illustrates that the bandwidth slow light with a group index of 118 can be obtained with an extremely low dispersion [14].



Fig. 5. Phase difference of MZI as a function of input light wavelength.

We set the lattice constant $a=336.7 \mathrm{~nm}$ to make the device operate at a low loss transmission wavelength of 1550nm. As is shown in Fig. 5, with the reflect center wavelength of FBG changes from $1550 \mathrm{~nm}$ to $1551 \mathrm{~nm}$, the phase difference of the MZI can be obtained according to Eq. (2). Moreover, as the reflect wavelength change of FBG is caused by a change of applied strain and the phase difference variation of the MZI would lead to the change of 
the output light intensity, we can get the relationship between the applied strain and the output intensity, which is shown in Fig. 6.

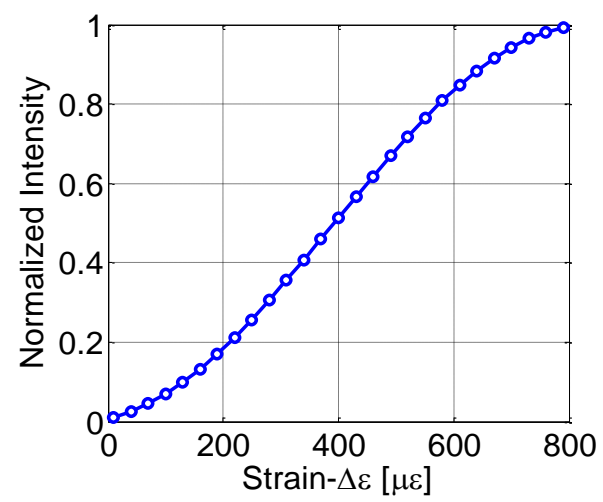

Fig. 6. Normalized output intensity of MZI as a function of applied strain.

From the simulation data we can get the sensitivity up to $3.6 \times 10^{-3} \mathrm{rad} / \mu \varepsilon$ with the MZI path mismatch is only $0.1 \mathrm{~mm}$. Because the MZI can be very short, therefore it is comparatively stable against external perturbations. The only concern is the thermal effect of silicon, which would cause the refractive index change of silicon. Hence an analysis of the temperature effect of PCW must be considered. Figure 7 shows the group index curves of our designed PCW when the working temperature $\mathrm{T}=290 \mathrm{~K}$ and $\mathrm{T}=300 \mathrm{~K}$ respectively. It is evident that the designed $\mathrm{PCW}$ possesses good temperature stability at the operation wavelength range from $1550 \mathrm{~nm}$ to $1551 \mathrm{~nm}$, which can guarantee the stable performance of the sensor for a desired temperature range.

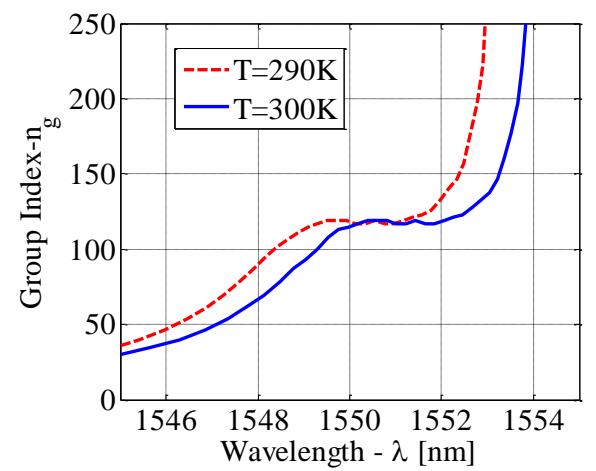

Fig.7. Group index of designed PCW as a function of wavelength for different temperatures.
In this paper, we present a novel high sensitivity slow light PCW based MZI configuration to monitor the reflect wavelength changes of an FBG sensor. The output intensity of MZI would be changed with a reflect center wavelength change of the FBG, which relates to the applied strain. With optimization of the structure of PCW, the sensitivity of this compact device is enhanced to 118 times of traditional MZI based strain sensors, that is about $3.6 \times 10^{-3} \mathrm{rad} / \mu \varepsilon$, benefiting from a high group index of 118 . Besides, the proposed device can eliminate any influence of temperature disturbance and can be widely used to measure strain, temperature, and other physical and chemical parameters with high sensitivity.

This work was partly supported by the National Natural Science Foundation of China (61203206, 61273059), the Fundamental Research Funds for the Central Universities (N100404006, N110204002, N110804003), and the Specialized Research Fund for the Doctoral Program of Higher Education of China (20120042120038), and the China Postdoctoral Science Foundation funded project (20110491511).

\section{References}

[1] M.H. Song, S.H. Yin, P.B. Ruffin, Appl. Opt. 39(7), 1106 (2000).

[2] S.M. Lee, S.S. Saini, M.Y. Jeong, Phot. Techn. Lett. 22(19), 1431 (2010).

[3] Y. Zhao, T.T. Song, Microwave Opt. Techn. Lett. 54(3), 822 (2012).

[4] S.J. Mihailov, Sensors 12(2), 1898 (2012).

[5] Y. Zhao, Y.N. Zhang, Q. Wang, Sensors and Actuators B: Chemical 160(1), 1288 (2011).

[6] M. Chahal, G.K. Celler, Y. Jaluria, Opt. Expr. 20(4), 4225 (2012).

[7] T. Baba, Nature Phot. 2(8), 465 (2008).

[8] C.Y. Shen, C. Zhong, Sensors and Actuators A: Physical 170(1-2), 51 (2011).

[9] http://ab-initio.mit.edu/wiki/index.php/MIT_Photonic_Bands.

[10] S.P. Guo, S. Albin, Opt. Expr. 11(2), 167 (2003).

[11] A.Y. Petrov, M. Eich, Appl. Phys. Lett. 85(21), 4866 (2004).

[12] J.T. Li, P. White, L.O. Faolain, Opt. Expr. 16(9), 6227 (2008).

[13] R. Hao, E. Cassan, X.L. Roux, D. Gao, V.D. Khanh, L. Vivien, M.M. Delphine, X.X. Zhang, Opt. Expr. 18(16), 16309 (2010).

[14] R.J.P. Engelen, Y. Sugimoto, Y. Watanabe, Opt. Expr. 14(4), 1658 (2006). 